\title{
The estimation of recurrence risks in monogenic disorders using flanking marker loci
}

\section{R M WINTER}

From the Kennedy-Galton Centre for Clinical Genetics, Harperbury Hospital, Radlett, Herts WD79HQ; and the Division of Inherited Metabolic Diseases, MRC Clinical Research Centre, Northwick Park Hospital, Watford Road, Harrow, Middlesex HA1 3UJ.

SUMMARY A method is presented of calculating recurrence risks at a disease locus where there is $:$ information from flanking marker loci. This method uses output from the computer programme $\overrightarrow{\mathrm{N}}$ LIPED. Information from carrier detection tests and mutation at the disease locus can be taken 응 into account in certain pedigrees.

Estimation of recurrence risks from pedigree data can be complex and prone to error. Complexity is further increased when ancillary tests, such as linked marker loci and carrier detection tests, are used. The present paper describes the use of LIPED, ${ }^{1}$ a generalised computer algorithm to estimate the likelihood of pedigrees segregating at a disease locus and at one marker locus, in order to estimate recurrence risks where there are flanking marker loci. It is hoped that this method will be useful as a check on calculations made by other means.

\section{Method}

The problem involves calculating the probability of a specific genotype at a disease locus $\left(d_{i}\right)$ in a consultand $\left(\right.$ Cons $\left.=d_{i}\right)$ given information about the disease status of members of the family (Dis) and information about phenotypes at two flanking marker loci $(\mathrm{A}, \mathrm{B})$ in the consultand and other family members. This probability can be expressed as:

$$
\left(\text { Cons }=\mathrm{d}_{\mathrm{i}} \mid \operatorname{Dis}, \mathrm{A}, \mathrm{B}\right)={\underset{\mathrm{j}}{\mathrm{i}=1}}_{\mathrm{j}\left(\text { Cons }=\mathrm{d}_{\mathrm{i}}, \text { Dis }, \mathrm{A}, \mathrm{B}\right)}
$$

where there are $\mathrm{j}$ possible genotypes at the disease locus. LIPED will give $\left(\right.$ Cons $\left.=d_{i}, D i s, A\right),($ Cons $=$ $d_{i}$, Dis, $\left.B\right)$, and (Cons $=d_{i}$, Dis) and it is necessary to derive (1) in terms of the above probabilities.

$\left(\right.$ Cons $=d_{i}$, Dis $\left., A, B,\right)=$ Cons $=d_{i}$, Dis $), \quad(A, B \mid$ Cons $=\mathrm{d}_{\mathrm{i}}$ Dis)

Received for publication 8 February 1984 Accepted for publication 27 July 1984.

$$
=\left(\text { Cons }=d_{i}, \text { Dis }\right) . \quad\left(A \mid \text { Cons }=d_{i}, \text { Dis }\right) .
$$

$\left(\mathrm{B} \mid\right.$ Cons $=\stackrel{\frac{0}{c}}{\stackrel{\frac{1}{2}}{2}}$

$d_{i}$, Dis) (2) providing (A Cons $=\mathrm{d}_{\mathrm{i}}$, Dis) is independent of $\vec{\bullet}$ $\left(B \mid\right.$ Cons $=d_{i}$, Dis $)$. This will be the case if there is no interference of crossing over. In other words, formula (2) can be used under the assumption that the coefficient of coincidence is one. ${ }^{2}$ Formula (2) can also be expressed as:

$$
\frac{\left(\text { Cons }=\mathrm{d}_{\mathrm{i}}, \text { Dis }, A\right)\left(\text { Cons }=\mathrm{d}_{\mathrm{i}}, \text { Dis }, \mathrm{B}\right)}{\left(\text { Cons }=\mathrm{d}_{\mathrm{i}}, \text { Dis }\right)}
$$

which can be used to calculate risks from formula (1) using LIPED, providing only persons whose genotype at the disease locus is known are used for calculating risks to the consultand and there is no. mutation at the disease locus. Where there are $\frac{}{3}$ persons in the family whose genotype at the disease locus is not known (for example, possible carrier females for an $\mathrm{X}$ linked disorder) formula (3) is calculated for each possible genotype of that person 을 and the sum of likelihoods is used.

\section{Carrier detection tests}

In certain $\mathrm{X}$ linked disorders, for example, $N$ Duchenne muscular dystrophy and haemophilia $A$, N carrier detection tests may be available on certain ${ }_{\sigma}^{\omega}$ females in the pedigree. In this case, formula (3) is still valid. The carrier detection results, expressed as a likelihood ratio given carrier or non-carrier status, can be used as a disease phenotype. Examples of this are given below.

\section{Mutation at the disease locus}

Unfortunately, using LIPED, calculation of recur- 
rence risks using flanking marker loci, where mutation is possible at the disease locus, is restricted to certain types of pedigree. These are (a) two generation pedigrees, where a possible mutation has occurred at the disease locus during gametogenesis in one of the parents; (b) three generation pedigrees, where persons in generation 2 have only one informative child.

Providing the above criteria are met, the likelihood of the pedigree, given that the parents of the possible new mutation are not carriers of the disease gene, can be calculated from the following formula:

$\left(\right.$ Cons $=d_{i}$, Dis $\left., A, B\right)=(A, B) .\left(\right.$ Cons $=d_{i}$, Dis $)(4)$ where $(A, B)$ represents the likelihood of the pedigree, considering markers $A$ and $B$ only, and the second expression is calculated allowing for mutation. Recurrence risks can be calculated, assuming equal mutation rates in males and females only. Examples are given below.

\section{Examples}

The examples all involve $\mathbf{X}$ linked disorders, but the general principles apply to any type of pedigree. Fig 1 shows a hypothetical pedigree for a lethal $\mathrm{X}$ linked disorder, such as Duchenne muscular dystrophy. Flanking probes (A and B) have been used which detect restriction fragment length polymorphisms with two alleles ( 1 and 2$)$ at each locus. The

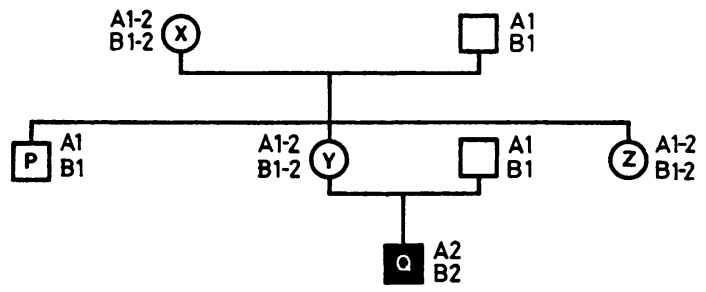

FIG 1 Pedigree for examples 1 to 3. recombination fraction between $A$ and the disease locus is 0.15 and between $B$ and the disease locus is $0 \cdot 1$. This means that, ignoring interference, the recombination fraction between $A$ and $B$ is $(0 \cdot 15+$ $0 \cdot 1)-(2 \times 0.15 \times 0.1)=0.22$. There are three possible female consultands in the pedigree, $\mathrm{X}, \mathrm{Y}$, and $\mathrm{Z}$.

Table 1 shows the results of four LIPED runs using every possible combination of carrier status for $\mathrm{X}, \mathrm{Y}$, and $\mathrm{Z}$. Some of these combinations are 'impossible' because they would involve two new mutations, and hence they are left blank. The figures given are the $\log _{10}$ of the pedigree likelihood (standard LIPED output). If $\mathrm{X}$ is a carrier, then there can be no new mutation in the pedigree and the likelihoods (Dis,A), (Dis,B), and (Dis) are calculated for the two possibilities of $Z$ being a carrier or not a carrier. (Dis,A,B) is calculated from formula (3).

If $\mathrm{X}$ is not a carrier, then the abnormal gene could have arisen in $Y$ or her affected son. $(A, B)$ using a recombination fraction between $A$ and $B$ of 0.22 and (Dis) (allowing for mutation) are calculated for these two possibilities and (Dis,A,B) is calculated using formula (4).

\section{EXAMPLE 1}

Probability $Z$ is a carrier if $X$ is an obligate carrier (fig 1)

In this case only the figures in sections $\mathrm{A}$ and $\mathrm{B}$ of table 1 need to be used.

$$
\begin{aligned}
(\mathrm{Z}=\text { carrier,Dis, } \mathrm{A}, \mathrm{B})= & \mathrm{Alog}_{10}(-7 \cdot 557325-7 \cdot 459815 \\
& +5 \cdot 861712) \\
= & \mathrm{A} \log _{10}(-9 \cdot 155428)=6.99152 \\
& \mathrm{E}-10 .
\end{aligned}
$$

Similarly $(\mathrm{Z}=$ non-carrier,Dis, $\mathrm{A}, \mathrm{B})=\mathrm{A} \log _{10}$

\begin{tabular}{|c|c|c|c|c|c|c|}
\hline & \multicolumn{3}{|l|}{$X$ carrier } & \multicolumn{3}{|c|}{$X$ non-carrier } \\
\hline & \multicolumn{2}{|l|}{$Y$ carrier } & \multirow[t]{2}{*}{$Y$ non-carrier } & \multicolumn{2}{|l|}{$Y$ carrier } & $Y$ non-carrier \\
\hline & \multicolumn{2}{|c|}{$\mathbf{A}$} & & \multirow{4}{*}{\multicolumn{2}{|c|}{-}} & \\
\hline \multirow[t]{3}{*}{$\mathrm{Z}$ carrier } & $(\mathrm{Dis}, \mathrm{A})(\overline{\mathrm{i}})$ & -7.557325 & & & & \\
\hline & (Dis,B)(ii) & -7.459815 & & & & \\
\hline & $\begin{array}{l}\text { (Dis)(iii) } \\
\text { (i)+(ii)-(iii) } \\
=(\text { Dis, A,B) }\end{array}$ & $\frac{-5 \cdot 861712}{-9 \cdot 155428}$ & - & & & - \\
\hline \multirow{5}{*}{$\mathrm{Z}$ non-carrier } & \multicolumn{2}{|l|}{ B } & \multirow{5}{*}{-} & \multicolumn{2}{|l|}{ C } & D \\
\hline & $(\mathrm{Dis}, \mathrm{A})(\mathrm{i})$ & $-8 \cdot 242451$ & & $(\mathrm{~A}, \mathrm{~B})(\mathrm{i})$ & $-4 \cdot 156418$ & $-4 \cdot 156418$ \\
\hline & (Dis,B)(ii) & $-8 \cdot 368895$ & & (Dis)(ii) & $-5 \cdot 269666$ & $-5 \cdot 259671$ \\
\hline & (Dis)(iii) & $\frac{-5 \cdot 861712}{10.710621}$ & & & & \\
\hline & $\begin{array}{l}\text { (i)+(ii)-(iii) } \\
=(\text { Dis, A,B })\end{array}$ & $-10 \cdot 749634$ & & $\begin{array}{l}\text { (i) }+(\text { ii) } \\
=(\text { Dis, } A, B)\end{array}$ & $-9 \cdot 416084$ & -9.416089 \\
\hline
\end{tabular}
$(-10.749634)=1.77977 \mathrm{E}-11$. So that the probability $\mathrm{Z}$ is a carrier, calculated from formula (1) is

TABLE 1 LIPED runs for examples 1 to 3. The figures given are $\log _{10}$ (likelihood). (See text for explanation.) 


$$
\frac{6.99152 \mathrm{E}-10}{(6.99152 \mathrm{E}-10+1.77977 \mathrm{E}-11)}
$$

This agrees with hand calculations.

\section{EXAMPLE 2}

Probability $X$ is a carrier, given there is no prior family history of the disorder (fig 1)

In this case the possibility of a new mutation at the disease locus has to be taken into account. The probability of the pedigree, given $X$ is not a carrier, must be calculated for the two possibilities that $\mathrm{Y}$ is or is not a carrier (sections $C$ and $D$ of table 1 ). In this case $(\mathrm{X}=$ non-carrier, $\mathrm{Y}=$ carrier,Dis, $\mathrm{A}, \mathrm{B})=$ $(\mathrm{A}, \mathrm{B})(\mathrm{X}=$ non-carrier, $\mathrm{Y}=$ carrier, $\mathrm{Dis})=\mathrm{Alog}_{10}$ $(-4 \cdot 156418-5 \cdot 269666)=A \log _{10}(-9 \cdot 416084)$ from formula (4).

Similarly $(\mathrm{X}=$ non-carrier, $\mathrm{Y}=$ non-carrier, Dis, $A, B)=A \log _{10}(-9 \cdot 416089)$. So the probability $\mathrm{X}$ is a carrier is given by

$$
A \log _{10}(-9 \cdot 155428)+A \log _{10}(-10 \cdot 749634)
$$

$$
\begin{gathered}
A \log _{10}(-9 \cdot 155428)+A \log _{10}(-10 \cdot 749634)+A \log _{10} \\
(-9 \cdot 416084)+A \log _{10}(-9 \cdot 416089)=0.4831
\end{gathered}
$$

This probability is calculated by hand from first principles in appendix 1.

\section{EXAM P L E 3}

Probability $Y$ is a carrier, if there is no other family history of the disorder (fig 1)

In this case mutation must be taken into account. The required probability is

$$
\begin{gathered}
A \log _{10}(-9 \cdot 155428)+A \log _{10}(10 \cdot 749634)+A \log _{10} \\
(-9 \cdot 416084) \\
A \log _{10}(-9 \cdot 155428)+A \log _{10}(-10 \cdot 749634)+A \log _{10} \\
(-9 \cdot 416084)+A \log _{10}(-9 \cdot 416089)=0 \cdot 7415
\end{gathered}
$$

EXA M P LE 4

Fig 2 shows another $\mathrm{X}$ linked pedigree. Marker loci are as for fig 1. Carrier detection tests have been carried out on $\mathrm{Y}$ and $\mathrm{Z}$. It is assumed that the carrier

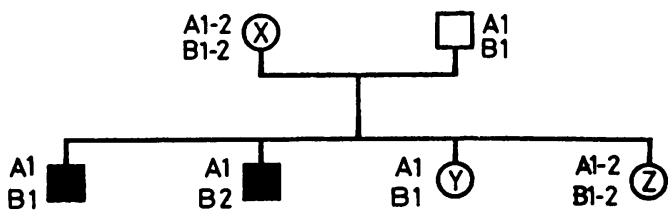

FIG 2 Pedigree for example 4.
TABLE 2 LIPED runs for example 4. (See text for explanation.)

\begin{tabular}{llrr}
\hline & & $Y$ carrier & $Y$ non-carrier \\
\hline Z carrier & (Dis,A)(i) & -9.299709 & -8.486572 \\
& (Dis,B)(ii) & $-10 \cdot 067861$ & -8.130566 \\
& (Dis)(iii) & $-7 \cdot 259647$ & -5.980893 \\
& (Dis,A,B)(i)+(ii)-(iii) & $-12 \cdot 107923$ & -10.636245 \\
Z non-carrier & (Dis,A)(i) & $-9 \cdot 161339$ & -8.623016 \\
& (Dis,B)(ii) & $-10 \cdot 011379$ & -9.391167 \\
& (Dis)(iii) & -7.861707 & -6.582953 \\
& (Dis,A,B)(i)+(ii)-(iii) & -11.311011 & -11.43123 \\
\hline
\end{tabular}

tests produce values $\mathrm{T} 1$ in $\mathrm{Y}$ and $\mathrm{T} 2$ in $\mathrm{Z}$ which give odds of being a carrier of $1: 19$ and $4: 1$ respectively. From this it can be seen that $\mathrm{T} 1$ is well into the 'normal' range and T2 is in the carrier range. Table 2 shows the results of LIPED runs, assuming $Y$ and $Z$ are or are not carriers. The probability $\mathrm{Z}$ is a carrier is given by

$$
A \log _{10}(-12 \cdot 107923)+A \log _{10}(-10 \cdot 636245)
$$

$A \log _{10}(-12 \cdot 107923)+A \log _{10}(-10 \cdot 6336245)+A \log _{10}$

$$
(-11 \cdot 311011)+\operatorname{Alog}_{10}(-11 \cdot 43123)=0.7355 \text {. }
$$

Appendix 2 shows this calculated by hand. Note that if the carrier detection tests on $\mathrm{Y}$ and $\mathrm{Z}$ were reversed the probability of $Z$ being a carrier would be 0.0029 , emphasising the importance of these tests in certain situations, even where flanking probes are available.

\section{Discussion}

The calculation of recurrence risks, using flanking marker loci, can present great difficulties, especially where carrier detection tests and mutation at the disease locus are a possibility. It is not claimed that the method presented in this paper is particularly convenient but, nevertheless, providing the few simple rules are followed, the procedure does appear to be reliable. Calculations of risks from first principles, even for relatively 'simple' pedigrees, as in the examples, can be exceedingly complex and prone to error. For this reason an independent check, using LIPED, can be invaluable. The restriction on pedigree structure, where mutation is a possibility, is a serious drawback. Purpose built computer programmes are being written to calculate recurrence risks using flanking marker loci. ${ }^{3}$ It is hoped that the method presented here will help to validate such programmes.

\footnotetext{
References

1 Ott J. A computer program for linkage analysis of general human pedigrees. Am J Med Genet 1976;28:528-9.
} , 
2 Elandt-Johnson RC. Probability models and statistical methods in genetics. New York: John Wiley, 1971:432-3.

${ }^{3}$ Lathrop GM, Lalouel JM. Easy calculations of Lod scores and genetic risks on small computers. Am J Hum Genet 1984;36:460-5.
Correspondence and requests for reprints to Dr R M Winter, The Kennedy-Galton Centre for Clinical Genetics, Harperbury Hospital, Harper Lane, Shenley, Radlett, Herts WD7 9HQ.

APPENDIX 1 Full derivation of the answer to example 2.

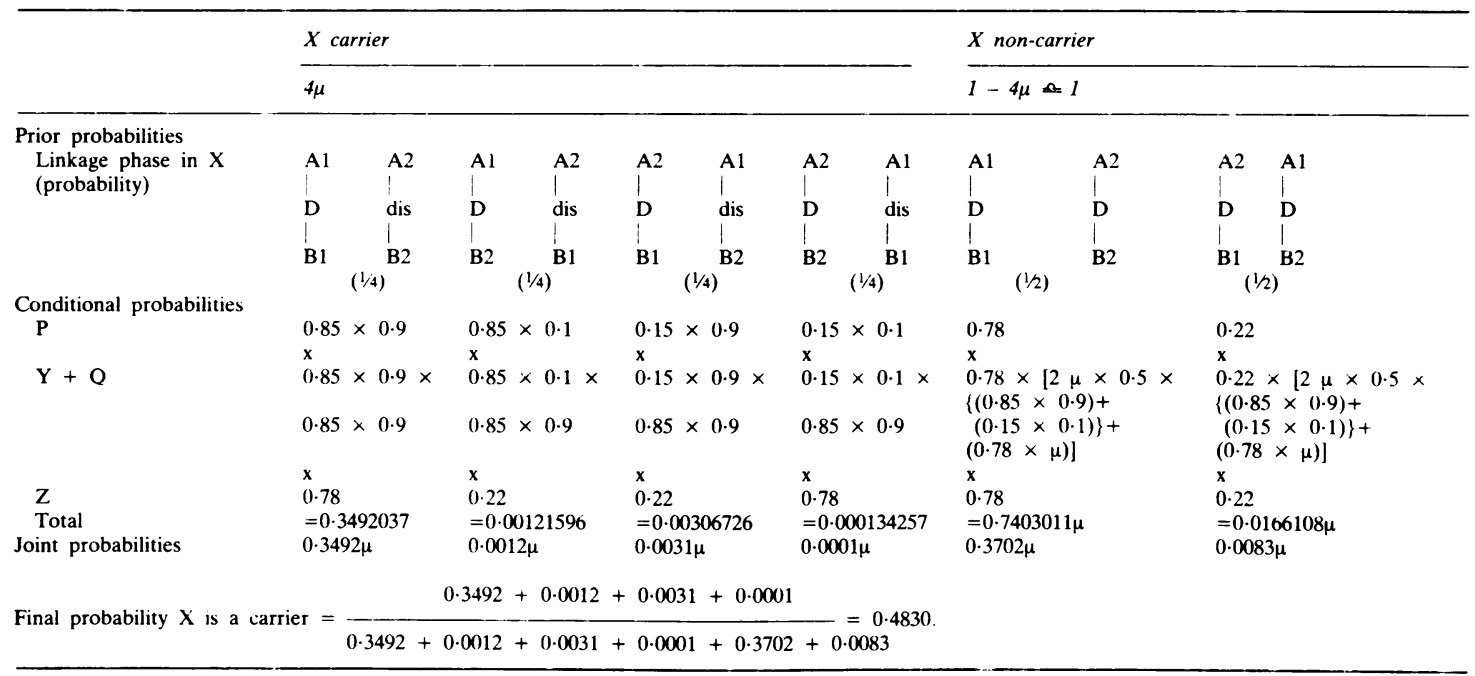

APPENDIX 2 Full derivation of the answer to example 4.

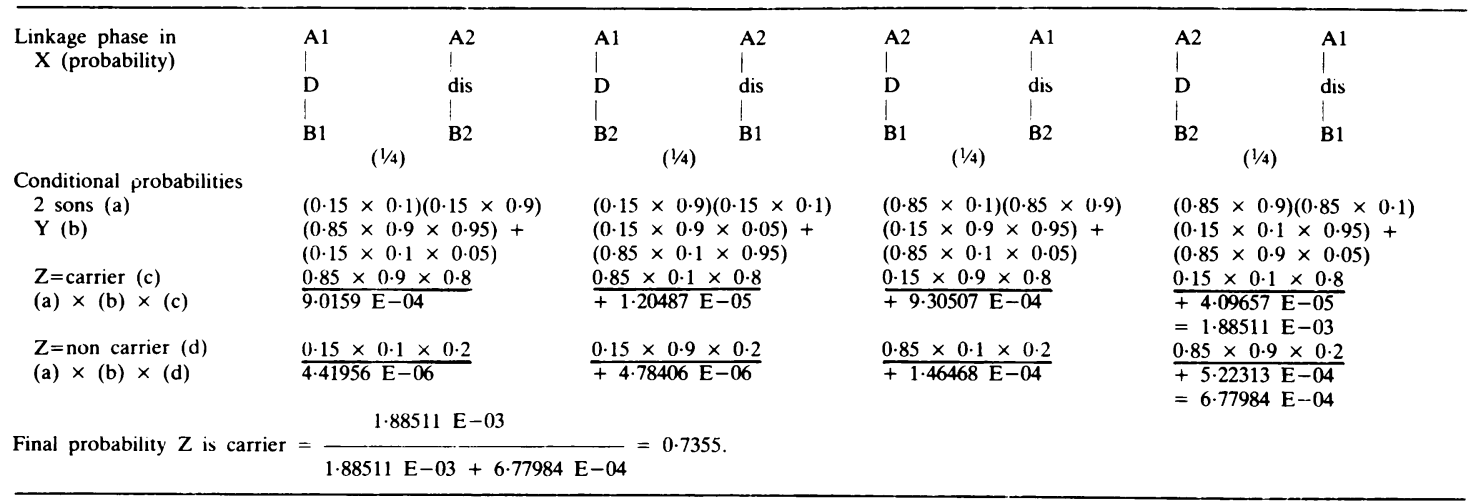

